ECONOMía. Teoría y Práctica • Nueva Época, número 46, enero-junio 2017,

pp. 97-113, http://dx.doi.org/10.24275/ETYPUAM/NE/462017/Ramirez

\title{
Una explicación de la rentabilidad y poder de mercado de las microempresas marginadas*
}

\section{Understanding the Mecanisms Behind the Profitability and Market Power of Marginalized Microenterprises}

\author{
Natanael Ramirez, ${ }^{* *}$ Alejandro Mungaray, ${ }^{* * *}$ \\ José G. Aguilar, ${ }^{* * * *}$ Ramón Inzunza****
}

\begin{abstract}
RESUMEN
El trabajo analiza el comportamiento de las microempresas bajo un marco de competencia imperfecta donde dichas unidades económicas son capaces de fijar un precio por encima de su costo marginal, el cual les permite subsistir e incluso ser rentables a pesar de las condiciones en las que suelen operar. Para probarlo, se estima un modelo econométrico que considera al índice de Lerner como variable dependiente de un conjunto de variables cualitativas previamente clasificadas por área de influencia. Se concluye que las microempresas son capaces de ser rentables y operar con poder de mercado gracias a la publicidad y estrategia de ventas utilizada, así como a la flexibilidad del proceso productivo. En cualquier caso, su capacidad de fijar precios está altamente influenciada por las condiciones socioeconómicas del mercado en el que operan.
\end{abstract}

Palabras clave: Microempresas, poder de mercado, marginación, índice de Lerner.

JEL: D4, D41, D21

\begin{abstract}
The paper analyzes the behavior of microenterprises in a context of imperfect competition in which they have the ability to profitably raise the market price of goods over their marginal cost. This allows them to survive and even be profitable despite the conditions under which they typically operate. We use an econometric model to conduct the analysis, in which we explain the Lerner index as a function of several qualitative variables previously classified by area of influence. We conclude that microenterprises are capable of being profitable and operating with market power through advertising and sales strategies due to the flexibility of the production process. In any case, the capacity to set prices is strongly influenced by the socioeconomic conditions of the market in which they operate.
\end{abstract}

Key words: Microenterprises, market power, marginalization, Lerner's Index.

JEL: D4, D41, D21

${ }^{*}$ Fecha de recepción: 22/08/2014. Fecha de aprobación: 23/05/2016.

** Universidad Autónoma de Baja California (UABC). Correo: natanael@uabc.edu.mx. ORCID: 0000-0002-4910-7648

*** Universidad Autónoma de Baja California (UABC). Correo: amungaray@uabc.edu.mx. ORCID: 0000-0001-5633-4585

**** Universidad Autónoma de Baja California (UABC). Correo: gaba@uabc.edu.mx. ORCID: 0000-0002-6378-6886

***** Universidad de la Sierra Sur de Oaxaca. Correo: riaxyzmx@hotmail.com. ORCID: 00000002-8675-6110 


\section{INTRODUCCIÓN}

En los años recientes, el análisis de las microempresas se ha posicionado como línea de investigación debido a la extendida participación de este sector en la base empresarial y su importante contribución al empleo. Diversos trabajos han demostrado la capacidad de las microempresas para absorber en tiempos de crisis el empleo que no retienen las medianas y grandes empresas (Georgellis, Sessions y Tsitsianis, 2005). Se ha demostrado también que son las empresas más pequeñas las que más capacidad tienen para ajustarse a los movimientos del mercado y por ello pueden atender una demanda potencialmente fluctuante de manera más eficiente (Mills y Schumann, 1985; Audretsch y Yamawaki, 1991).

A pesar de su importancia, el análisis sobre el comportamiento competitivo de estas unidades económicas y los mecanismos implementados para su sobrevivencia en el mercado son escasos (Palacio, 2002). Tradicionalmente se ha asumido que por ser "muchas" y por contar con escalas de producción "pequeñas", el marco de competencia que rige su comportamiento es el de competencia perfecta. Sin embargo, diversos estudios han demostrado que el tamaño de la empresa no es condicionante del grado de poder de mercado con el que opera, ya que la diferenciación del producto o la ubicación geográfica, entre otros, pueden permitir que incluso pequeñas empresas puedan tener precios significativamente por encima de sus costos (Shama, 1993; Barla, 2000; Vázquez, 2005). Trabajos empíricos han demostrado que la microempresa que surge en zonas periféricas de las ciudades grandes puede operar con poder de mercado en función de la diferenciación que logre gracias a su ubicación geográfica en zonas de marginación, captando así una demanda que tiene poco interés para las medianas y grandes empresas debido a los costos de transacción que implica llegar a los consumidores constituidos en pequeños nichos de barrio (Mungaray et al., 2005).

El objetivo del presente trabajo es determinar los factores que permiten que las microempresas marginadas, ${ }^{1}$ a pesar de las condiciones técnicas en las que operan, puedan fijar precios por encima de sus costos marginales, ejerciendo así, poder de mercado. El trabajo sostiene la hipótesis de que microempresas marginadas pueden operar con poder de mercado si consiguen diferenciarse a través de acciones relacionadas con la mejora del producto, la atención al cliente o el aprovechamiento de las cualidades del punto de venta por sus condiciones geográficas. El trabajo se

\footnotetext{
${ }^{1}$ Para este trabajo, microempresa marginada se refiere a una unidad económica que no cuenta con más de cinco trabajadores, operan con tecnología tradicional, baja escolaridad de los trabajadores y del propietario y se encuentran ubicadas en las zonas de mayor nivel de marginación de la ciudad.
} 
organiza como sigue: en el primer apartado se realiza una revisión de la literatura referente al poder de mercado presente en microempresas; posteriormente se describe la fuente de información y se plantea un modelo empírico que permite medir cuáles son las variables de mayor incidencia en la transferencia de poder de mercado a las microempresas; en el segundo apartado se discuten los resultados en el marco del debate actual; y, finalmente, en la sección tercera se presentan las principales conclusiones y limitaciones del trabajo.

\section{Poder de Mercado En Microempresas}

El poder de mercado para una empresa individual se conoce como la capacidad o facultad que tiene ésta para influir sobre el precio del bien o servicio que ofrece para la venta (Parkin y Esquivel, 2006; White, 2013). Para Stoft (2002) el poder de mercado implica la capacidad de alterar, en beneficio propio, el precio respecto a los niveles de competencia, el cual puede surgir por la diferenciación que se puede hacer del producto o de la venta del mismo (White, 2013). De acuerdo con el clásico paradigma Estructura-Conducta-Resultados (ECR) de Chamberlin (1933), cuando el producto carece de una intensa competencia, existen pocas empresas en el mercado y éstas tienen una participación significativa en las ventas totales, facilitando la aparición de conductas monopólicas que influyen en la fijación de un precio por encima de costos, provocando una reducción del bienestar social (Sylos, 1966; Fariñas y Huergo, 2003). A esto se asocia el poder de mercado de la empresa que resulta de una competencia que elimina o absorbe a otros y que facilita la entrada de nuevos (Clarke, 2000).

Esta definición considera que son sólo las grandes las que, al concentrar la mayor parte de las ventas del mercado, pueden ejercer prácticas exitosamente asociadas del poder de mercado. Investigando si esta capacidad también está asociada a la presencia de otras variables como el grado de diferenciación del producto, la ubicación geográfica de la empresa o la lealtad de los consumidores, en su teoría de localización, Hotelling (1929) plantea que la ubicación geográfica podría concederle poder de mercado a la empresa en presencia de bienes homogéneos o sustitutos cercanos, pues los consumidores cuya localización sea cercana a una de las empresas, deberán incurrir en un "alto" costo de transacción para comprar en una empresa competidora. Esto puede ser aprovechado por las empresas cercanas, las cuales podrían incrementar moderadamente sus precios sin que la pérdida de clientes sea significativa. Esta situación es muy frecuente en el sector microempresarial de unidades comerciales ubicadas en zonas periféricas o con algún tipo de marginación, las cuales, a través de la atención de nichos de barrio, logran sobrevivir y ser rentables económicamente (Mungaray et al., 2005). De acuerdo con este 
planteamiento, entre mayor sea el nivel de marginación, la microempresa tendrá mayor capacidad para actuar con poder de mercado, teniendo como límite su capacidad productiva y de gestión y la restricción presupuestaria de los clientes. Sin embargo, aún queda mucho por investigar acerca de si la marginación social y económica son condición necesaria o suficiente para la aparición de poder de mercado en una microempresa con limitaciones de operación.

Estudios como los de Davies y colaboradores (1984), Daniels (1999) y Mungaray y colaboradores (2007), muestran que las microempresas establecidas en las zonas económicamente más vulnerables son capaces de construir un grado de diferenciación que les permite fijar precios por encima de costos marginales. Por otra parte, Orlando y Pollack (2000) encuentran que cuando las microempresas surgidas en las zonas pobres de muchos países de América Latina logran sobrevivir algunos años, se debe a la protección derivada de la propia situación de marginación del entorno, que las hace aún más estables que otras de barrios con mayor poder adquisitivo.

A pesar de que existen avances importantes en la identificación y medición de la capacidad de las micro y pequeñas empresas para operar con poder de mercado, de acuerdo con Bresnahan (1989), la literatura no presenta argumentos contundentes acerca de su naturaleza y relación con otros factores tales como la tecnología utilizada, la calidad del producto, la lealtad de los consumidores o la formación del microempresario. Por ejemplo, es natural que en toda industria haya empresas más eficientes que otras ya sea por contar con una mejor tecnología, más años en el mercado o una mejor dirección (Demsetz, 1974), y que las empresas grandes intenten mejorar su posición competitiva a través de estos elementos de la innovación y diferenciación.

Por otra parte, el estudio de López, Contreras y Molina (2013) demuestra que la capacidad de crear innovaciones o diferenciarse no es exclusivo de las grandes empresas, debido a que la planeación, si bien es poco común en las pequeñas empresas, se presenta con las estrategias de competitividad que realizan, y que incluso pueden ser más continuas que sus homólogos más grandes. En este sentido, Landa (2013) comenta que existe una relación directa entre la flexibilidad en la producción y la creación de innovaciones en las actividades de la empresa, por lo que tomando que el ser flexible es característico de la microempresa, ésta podría ser prueba de que generan poder de mercado utilizando dicha característica. De igual manera, la diferenciación es parte de las ventajas de la microempresa debido a que pueden adaptarse con facilidad a los diferentes clientes que quedan excluidos de la atención de otras empresas, ya sea porque no están dentro de los objetivos de la misma o porque no poseen la flexibilidad necesaria para realizarlo (Guarneros, 2013). 
La teoría de capital humano señala que algunos atributos inherentes a los trabajadores, como la educación y el estado de salud, pueden ser considerados como factores que pueden traer beneficios o perjuicios económicos a las organizaciones (Schultz, 1960 y 1971). En este sentido, el aumento en el nivel de formación, como una estrategia para fortalecer las habilidades cognitivas del dueño y los trabajadores, impulsa la productividad de los trabajadores y fortalece las habilidades empresariales, lo cual es un factor condicionante para el ejercicio de poder de mercado (Mungaray et al., 2007). Independiente del tamaño de la organización, el control de los diferentes recursos es necesaria para la eficiencia de la empresa, por lo que la capacitación y asesoría constante, como parte de la formación de los trabajadores, es necesaria sobre todo en sectores más desprotegidos (Almaraz, 2013). Por otra parte, cuando se trata de microempresas, la falta de salud del promotor es la antesala del término de la aventura empresarial, por lo que si consideramos que un gran número de microunidades mexicanas está dirigida por amas de casa, la productividad puede verse afectada porque la mujer empresaria no se desentiende de sus labores y compromisos familiares, aun desempeñando una actividad productiva (Parada, 2013).

Borch y Forsman (1999) consideran tres estrategias de posicionamiento en el mercado para las empresas: la diferenciación del cliente mediante la segmentación basada en criterios como la edad, el nivel de educación, el nivel de ingresos y el estrato social, entre otros; la diferenciación del producto, que considera distintas dimensiones de la calidad, las modas y los gustos; y la diferenciación por publicidad, la cual, a través de la mercadotecnia, permite ajustar en el mediano plazo las preferencias de los consumidores. Si bien estas estrategias facilitan la manipulación del nivel de precios, lo cierto es que en las empresas más pequeñas estas oportunidades son muy limitadas. Sin embargo, empresas como Ford, McDonalds y Wall Mart presentaron en sus inicios las mismas limitantes de mercado que las pequeñas empresas (Gross, 2011).

Por otra parte, el posicionamiento en los canales de distribución del producto permite ampliar las dimensiones del mercado y disminuir costos en empresas medianas y grandes a través de la subcontratación. Esta alternativa comercial provee certidumbre con respecto al nivel de ventas y permite una planeación de los niveles de ingreso, gasto, inventarios e inversión (Mungaray y Ramírez, 2004). Aunado a la distribución del producto, el lugar donde se establece la microempresa puede considerarse una variable importante, sobre todo al inicio de operaciones, ya que una mala elección podría representar una fuerte barrera para la evolución de la empresa (Audretsch, 2001) debido a la insuficiencia de capital para tomar acciones correctivas. 


\section{Metodología}

Autores como Davies y colaboradores (1984), Daniels, (1999) y Mungaray y colaboradores (2007) coinciden en las variables que de forma individual y combinada pueden favorecer que unas cuantas empresas controlen un mercado cuando sus niveles son adecuados para sobresalir en un entorno de competencia específico. Algunas de estas variables son: el grado de adaptación del producto y de empatía con las necesidades de clientes, el tipo de tecnología empleada, el gasto en publicidad, los canales de distribución y puntos de venta creados, la longevidad de la empresa, el nivel de educación del dueño y de los trabajadores, la productividad de estos empleados y la infraestructura y servicios en el entorno en el que se localiza la empresa.

Las variables se pueden clasificar en cuatro grupos según esté relacionada con las cualidades del producto, las cualidades de los trabajadores, las cualidades del establecimiento y las cualidades del mercado. Dentro de las cualidades del producto, algunas estrategias se refieren a mejorar la calidad y a buscar la diversificación y adaptación para cubrir mejor las necesidades de los clientes. Dentro de las cualidades de la fuerza de trabajo, se puede considerar como determinantes el nivel de formación del dueño y empleados, el número de trabajadores, así como su productividad. En las cualidades del negocio hay que incluir el tipo de publicidad utilizado, el manejo de los canales de distribución y puntos de venta, las posibilidades de implementación de la opción de la subcontratación y la longevidad de la empresa. En cuanto a las cualidades del mercado, ante la ausencia de información acerca de la opinión directa de los consumidores, el índice de marginación puede funcionar como una variable proxy de las condiciones socioeconómicas de los clientes atendidos por la microempresa.

\section{II.1. Modelo econométrico}

Existen diferentes modelos para medir el poder de mercado y sus efectos en el óptimo de bienestar, algunos de ellos son: análisis de regresión con sección cruzada; estudios de concentración de precios; datos de subasta; análisis de la $q$ de Tobin; el enfoque Panza-Rosee; y el enfoque de la nueva organización industrial empírica (Bikker, Sherrill y Spierdijk, 2012; White, 2013). Todos ellos requieren de la disponibilidad de datos microeconómicos de las empresas, que en muchas ocasiones resulta difícil obtener. Para este trabajo se utiliza el modelo de regresión de sección cruzada que se estima por medio de mínimos cuadrados ordinarios y se deriva del utilizado por Mungaray y colaboradores (2007), en el cual se asume que cada 
microempresa se encuentra operando en un entorno de competencia imperfecta y que la aparición de poder de mercado es posible. Se considera como variable dependiente al índice de Lerner (1934), el cual proporciona una medida relativa del poder de mercado de una empresa. Este índice se estima mediante la siguiente ecuación:

$$
L_{i}=\left(\frac{P_{i}-C m g_{i}}{P_{i}}\right)
$$

donde el índice de Lerner para la empresa $i$ se denota por $L_{i}$; el precio de su producto es representado por $P_{i}$, y su costo marginal se denota por $C m g_{i}$. Huergo (2001) menciona que dada la inobservancia del costo marginal, una buena aproximación para $\mathrm{Cmg}_{i}$ puede ser el costo medio de producción del bien $\left(\mathrm{Cme}_{i}\right)$, una relación usualmente aceptada, antes de que Lerner incorporara la diferencia entre el precio y costo marginal para medir la pérdida social en un monopolio (Elzing y Mills, 2011). Las implicaciones negativas de la aproximación anterior son mínimas comparadas con los beneficios que aporta, sobre todo cuando se trata de estudios de corte transversal como en este caso. De esta forma, el índice de Lerner en términos funcionales se puede replantear como:

$$
L_{i}=\left(\frac{P_{i}-C m e_{i}}{P_{i}}\right)
$$

Este índice tiene el potencial de medir la capacidad de la empresa por mantener demanda positiva aun con aumentos en el precio. El modelo mide la relación del índice de Lerner con grupos de variables cualitativas asociadas a las características del establecimiento, trabajadores, producto y mercado.

\section{II.2. Construyendo el índice de marginación}

De acuerdo con el Consejo Nacional de Población (Conapo), la marginación es un fenómeno estructural originado en el patrón histórico de desarrollo. Ésta se expresa en la dificultad para propagar el progreso técnico en el conjunto de la estructura productiva local y en la exclusión del proceso de desarrollo y del disfrute de sus beneficios para ciertos grupos sociales. Para la construcción de un indicador del grado de marginación, se considera la intensidad espacial de nueve manifestaciones de la exclusión, medidas por los porcentajes de población para quienes el disfrute de ciertos bienes y servicios esenciales para el desarrollo no es posible.

Estos elementos de marginación, los cuales están asociados a la vivienda, son los siguientes indicadores expresados en porcentaje respecto al total de la población: 
ocupantes en viviendas particulares sin agua entubada; ocupantes en viviendas $\sin$ drenaje ni sanitario exclusivo; ocupantes en viviendas sin energía eléctrica; ocupantes en viviendas con piso de tierra; viviendas con niveles significativos de hacinamiento; población ocupada con ingresos de hasta dos salarios mínimos; población de 15 años o más que es analfabeta y población de 15 años o más sin primaria completa; y población que vive en localidades de menos de 5,000 habitantes.

Para la estimación del índice de marginación se consideró como fuente de información el Censo de Población y Vivienda de 2010, debido a que cuenta con la cobertura y grado de desagregación necesarios para su análisis a nivel estatal y municipal. El índice de marginación por zona geográfica fue construido con base en la metodología utilizada por la Conapo en su índice de marginación estatal y municipal en México en el año 2000, para la cual se utiliza la técnica de componentes principales con base en la siguiente ecuación:

$$
I M_{j}=\sum_{i=1}^{w} F P_{i} \frac{\left(X_{i, j}-\bar{X}_{i}\right)}{d_{i}}
$$

donde $I M_{j}$ es el índice de marginación de la unidad geográfica $j$. Además, para el indicador $i, X_{i, j}$ es el valor que toma en la unidad geográfica $j, \bar{X}_{i}$, es su media, $d_{i}$ es su desviación estándar y $F P_{i}$ es su factor de ponderación. El total de indicadores de marginación utilizados es $w$.

Considerando el índice de Lerner y el de marginación descritos en las ecuaciones 2 y 3 respectivamente, y variables cualitativas sobre las cualidades del establecimiento, de los trabajadores, del producto y del mercado, el modelo a estimar se representa por la siguiente ecuación:

$$
\begin{aligned}
L_{i}= & \beta_{0}+\beta_{1} A O_{i}+\beta_{2} P B_{i}+\beta_{3} L V_{i}+\beta_{4} E V_{i}+\beta_{5} A D_{i}+\beta_{6} N T_{i} \\
& +\beta_{7} I P_{i}+\beta_{8} M P_{i}+\beta_{9} D P_{i}+\beta_{10} A P_{i}+\beta_{11} I M_{i}+\mu_{i}
\end{aligned}
$$

donde, particularizado para la microempresa $i$, la variable $A O_{i}$ representa los años de operación; $P B_{i}$ representa el grado de publicidad que consiste en una dummy que toma el valor de 1 si la microempresa está aplicando alguna estrategia de publicidad y 0 en caso contrario; el lugar de venta está representado por la expresión $L V_{i}$, que toma el valor 1 si la microempresa vende en ambulantaje, 2 cuando el puesto está ubicado en la vía pública de forma estable, 3 si la microempresa tiene un puesto de venta en casa propia, y 4 si está situada en un local establecido e independiente del hogar; $E V_{i}$ se refiere a la existencia de estrategias de venta, en cuyo caso toma valor de 1 cuando aplica alguna y de 0 cuando no lo hace; $A D_{i}$ se refiere a la adaptación o remodelación del establecimiento que implique ajustes en 
el proceso productivo y toma el valor de 1 cuando esto se ha hecho durante el último año y 0 cuando no ha ocurrido así.

$N T_{i}$ representa el número de trabajadores; $I P_{i}$ es una variable proxy de la productividad que se calcula dividiendo el valor de la producción mensual entre el número de trabajadores. Las variables $M P_{i}, D P_{i}$ y $A P_{i}$ capturan efectos de cambios en el producto para mejorarlo, diferenciarlo o adaptarlo respectivamente, con variables dummies que toman valor de 1 cuando ocurrieron los cambios y 0 en cualquier otro caso. El índice de marginación de la colonia donde se ubica la microempresa $i$ está dado por $I M_{i}$. Por último, $\mu_{i}$ representa los errores del modelo y los parámetros $\beta_{1}, \ldots, \beta_{11}$ son los coeficientes que miden el nivel de relación entre el índice de Lerner y las variables explicativas descritas previamente.

\section{II.3. Fuentes de información}

La información se obtuvo del Programa de Investigación, Asistencia y Docencia a la Micro y Pequeña Empresa (PIADMyPE) de la Universidad Autónoma de Baja California, el cual brinda asistencia técnica en materia de administración, producción, costos $\mathrm{y}$ aspectos fiscales a microempresas marginadas del estado de Baja California. A través de instrumentos como la encuesta diagnóstico y la bitácora de operaciones, este programa obtiene información para dar seguimiento a las microempresas atendidas. El PIADMyPE se ha aplicado desde el 2002 hasta el 2015, buscando impulsar el desarrollo de microempresas para favorecer su crecimiento y mejorar el nivel de bienestar de las familias que dependen de estas unidades académicas.

Para el ejercicio empírico se seleccionó una muestra aleatoria de 1,323 microempresas que fueron atendidas de 2008 al 2012, que operan en las colonias con los mayores niveles de pobreza y marginación de la entidad, donde además el nivel de formación del microempresario es limitado. Las microempresas consideradas en la muestra desarrollan actividades dentro de los sectores comercio, manufactura y servicios. En el cuadro 1 se aprecia que con excepción del municipio de Tecate, el sector principal es el comercial, el cual tiene una representatividad agregada del 43.7 por ciento.

En este ambiente de marginación, el 66 por ciento de las microempresas analizadas está a cargo de una mujer, lo cual es consecuencia de que el surgimiento de la mayoría de este tipo de microempresas está muy ligado a un clima familiar asociado a la precariedad e insuficiencia de recursos económicos. En países en vías de desarrollo, la decisión de abrir una microempresa requerirá además del aumento de la capacidad de negociación en el hogar por parte de la mujer (Pollack, 2005), 
lo cual hace posible que sean las amas de casa las que decidan principalmente abrir un negocio de estas características.

Cuadro 1. Descripción de la muestra

\begin{tabular}{lccccccccc}
\hline & \multicolumn{10}{c}{ Municipio } \\
\cline { 2 - 10 } Sector & $\begin{array}{c}\text { Ensenada } \\
(13 \%)\end{array}$ & $\%$ & $\begin{array}{c}\text { Mexicali } \\
(-43 \%)\end{array}$ & $\begin{array}{c}\text { Tecate } \\
(3 \%)\end{array}$ & $\begin{array}{c}\text { Tijuana } \\
(40 \%)\end{array}$ & $\%$ & $\begin{array}{c}\text { Total } \\
(100 \%)\end{array}$ \\
\hline Comercio & 64 & 38 & 218 & 38 & 19 & 41 & 277 & $52 \%$ & 578 \\
Servicios & 55 & 32 & 210 & 37 & 23 & 50 & 157 & $29 \%$ & 445 \\
Manufactura & 51 & 30 & 144 & 25 & 4 & 9 & 101 & $19 \%$ & 300 \\
Total & 170 & 100 & 572 & 100 & 46 & 100 & 535 & $100 \%$ & 1,323 \\
\hline
\end{tabular}

Fuente: Elaboración propia con datos del PIADMyPE.

El promedio de trabajadores es de 1.4 incluyendo el dueño, en el caso de contar con un trabajador adicional es algún miembro de la familia; el promedio de antigüedad es de 1.4 años y las ventas promedio mensual son de $\$ 16,403$, con diferencias importantes entre municipios, donde en las ciudades más grandes tiende a ser menor, quizás por la mayor concentración de microempresa aún en las colonias de mayor marginación. Muchas de estas microempresas surgen ante la imposibilidad de encontrar un empleo en el mercado laboral o en el caso de las mujeres, por la necesidad de estar cerca de sus hogares y atender el resto de las necesidades familiares.

Cuadro 2. Características de las microempresas

\begin{tabular}{lcccccccc}
\hline & \multicolumn{7}{c}{ Variables } \\
\cline { 2 - 8 } Municipio & $\begin{array}{c}\text { No. } \\
\text { Trabajadores }\end{array}$ & $\begin{array}{c}\text { Ventas } \\
\text { mensuales }\end{array}$ & Antigüedad & Hombres & $\%$ & Mujeres & $\%$ & Total \\
\hline Mexicali & 1.4 & $\$ 12,855$ & 1.6 años & 216 & 38 & 356 & 62 & 572 \\
Tecate & 1.4 & $\$ 26,719$ & 0.8 años & 11 & 24 & 35 & 76 & 46 \\
Tijuana & 1.5 & $\$ 10,434$ & 1.8 años & 160 & 30 & 375 & 70 & 535 \\
Ensenada & 1.3 & $\$ 15,606$ & 1.3 años & 61 & 36 & 109 & 64 & 170 \\
Total & 1.4 & $\$ 16,403.50$ & 1.4 años & 448 & 34 & 875 & 66 & 1,323 \\
\hline
\end{tabular}

Fuente: Elaboración propia con datos del PIADMyPE. 


\section{AnÁlisis de RESUltados}

Los resultados de la estimación de la ecuación 4 se presentan en el cuadro 3 y muestran que únicamente cuatro de las 11 variables explicativas fueron estadísticamente significativas al 90 por ciento de confianza o superior, por lo que la hipótesis planteada se confirma sólo parcialmente. El hecho de que los resultados arrojan un $R^{2}$ bajo para ser una estimación con minímos cuadrados ordinarios, lo cual evidencia cierta dificultad para predecir resultados individuales precisos, no implica ausencia del efecto de las variables en aquellas que fueron estadísticamente signficativas.

Tres de las variables con capacidad de explicar el poder de mercado en microempresas ubicadas en zonas de marginación pertenecen a las cualidades del establecimiento, como el tipo de publicidad, la estrategia de ventas que se implementan y los ajustes que se hacen al local para adecuarlo a las necesidades del cliente. Esto permite afirmar que la cercanía al cliente y la lealtad que pueda desarrollarse otorgan al microempresario cierto poder para incrementar el precio. Sin embargo, los resultados indican que para este tipo de empresas la antigüedad y ubicación del negocio por sí mismos no otorgan poder de mercado.

Para Parkin y Esquivel (2006) el impulso de la publicidad es importante para comunicar la calidad del producto que se ofrece o comprometerse con el consumidor en una calidad mínima o persuadirlo a través de la presentación. A pesar de su importancia, esta es una actividad poco valorada y explotada por los microempresarios debido a la falta de recursos económicos para pagar el diseño de un plan estratégico de marketing o la falta de capacitación para hacerlo ellos mismos, incluyendo la limitada utilización de tecnologías de información cada vez más comunes para la difusión de los productos y servicios. Este resultado representa una gran oportunidad para mejorar la situación competitiva de la microempresa a través de la exaltación de las cualidades del producto, sobre todo cuando se trate de bienes artesanales, en contra de aquellos que son resultado del trabajo industrializado, ya sea desde el ámbito de la salud (en el caso de alimentos) o de lo artístico (en el caso de artículos ornamentales).

Contar con una estrategia de venta encaminada a un mejor servicio presenta una relación positiva con la variable dependiente. No obstante, a pesar de su importancia, sólo 40 por ciento de las microempresas manifestaron utilizar algún tipo de estrategia para mejorar sus ventas. Vázquez (2005) plantea que las relaciones con microempresarios a través de asociaciones, como las realizadas con clientes a través de estudios de mercado, favorecen el surgimiento de innovaciones que crean ventajas competitivas, convirtiendo la estrategia de atención al cliente en piedra angular para el éxito del micronegocio. Dentro de las estrategias convencionales de servicio al cliente se encuentran el trato cercano, la entrega oportuna y el servicio a domicilio. 
Los hallazgos relacionados con la adaptación de los procesos o del establecimiento ante las necesidades cambiantes de los clientes coincide con los resultados clásicos de Borch y Forsman (1999), en el sentido de que una continua adaptación de la microempresa para lograr segmentar a los clientes y actuar con poder de mercado permite que el consumidor se sienta tomado en cuenta y tenga confianza en que el establecimiento se ajustará a sus recomendaciones. Esto podría otorgar ventaja respecto a las cadenas de supermercados y de autoservicios que son menos flexibles debido a la heterogeneidad de su mercado, y más lentas en su toma de decisiones y capacidad de reacción.

La antigüedad de las empresas no presenta relación con la variable dependiente debido a que la naturaleza de estas microempresas se asocia a la supervivencia económica, donde no está claramente definida la barrera entre la administración de la microempresa y la gestión familiar, inhibiéndose su crecimiento. En el caso específico de su ubicación, los resultados indican que, para las microempresas establecidas en áreas de alta marginación, el grado de formalidad, independencia y estabilidad del local comercial no dan mayor capacidad de poder de mercado al microempresario. Aunque un local independiente y estable permitirá que el cliente se sienta identificado con la microempresa — ante la ausencia de branding -, los resultados sugieren que el microempresario de naturaleza informal utiliza otros instrumentos valorados por los clientes potenciales para crear confianza, aun si su proyecto es de venta ambulante.

En lo que concierne a las cualidades de los trabajadores, ni el número de empleados por sí mismo o la productividad de éstos se asocian al poder de mercado, lo cual se puede explicar por el bajo nivel de formación que tienen los trabajadores y propietarios de este tipo de establecimientos. Las cualidades del producto tampoco mostraron una influencia clara sobre la capacidad de poder de mercado de las microempresas surgidas en entornos de marginación. En el caso de la diversificación del producto, la falta de relación podría estar asociada al hecho de que este tipo de empresas son de naturaleza artesanal, haciendo complejo para el consumidor identificar una estrategia de diversificación más allá de la propia atención personalizada por parte del personal de la microempresa (Borch y Forsman, 2004).

Finalmente, el ambiente de mercado medido por el índice de marginación muestra una relación positiva con el índice de Lerner. Lo anterior apoya hallazgos presentados en trabajos previos como el de Mungaray y colaboradores (2007), donde se argumenta que las zonas de marginación en las que se localizan las microempresas, las cuales presentan altos niveles de inseguridad y limitaciones de infraestructura y comunicaciones, ofrecen una protección natural ante la entrada de nuevos competidores, dándoles a las ya establecidas la capacidad de manejar por lo que dependerá del microempresario transformar esta oportunidad en una 


\section{Cuadro 3. Resultados econométricos}

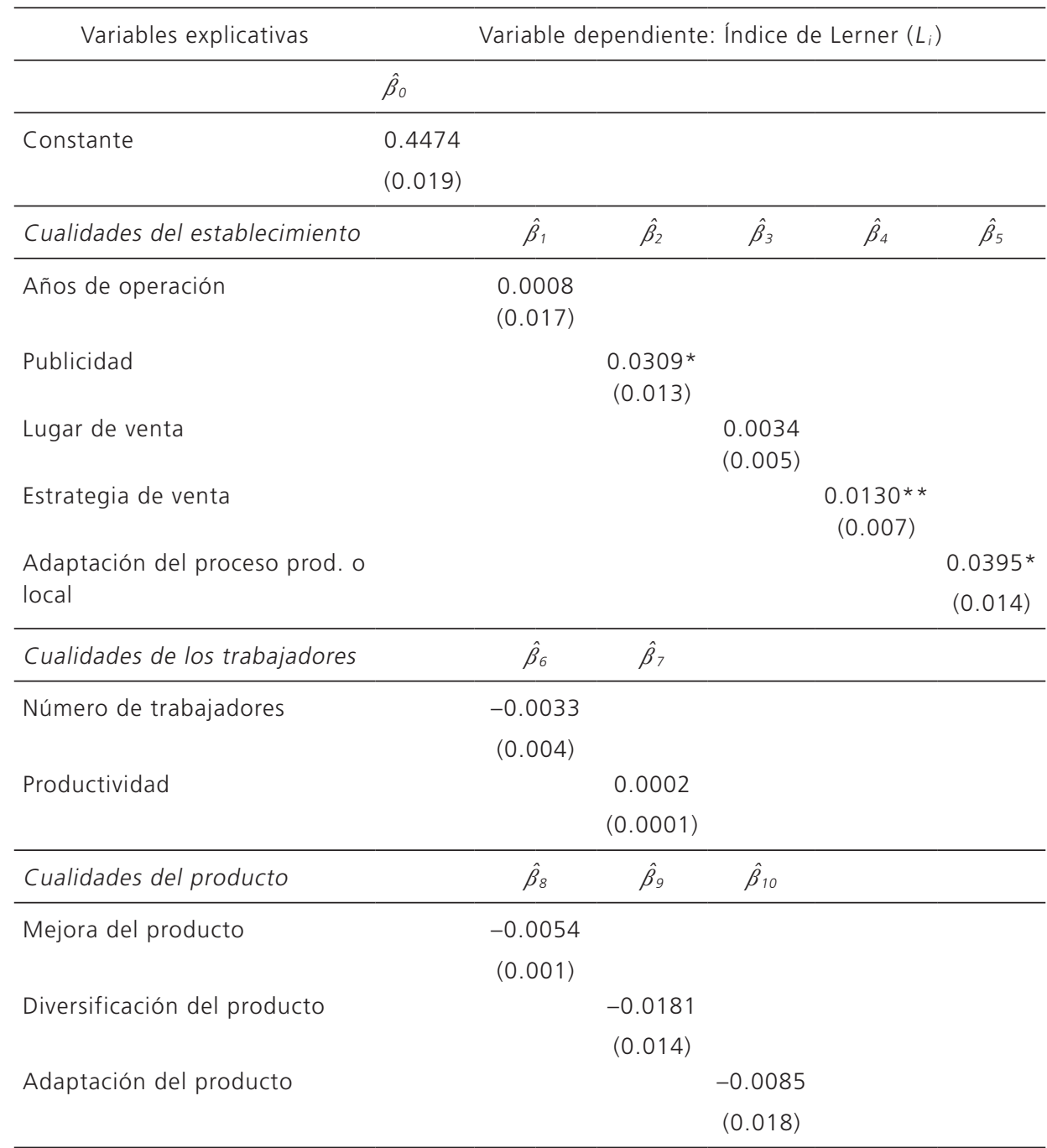

\begin{tabular}{lc}
\hline Ambiente del mercado & $\hat{\beta}_{11}$ \\
\hline Índice de marginación & $0.0018^{* *}$ \\
& $(0.001)$ \\
\hline
\end{tabular}

R2 $=0.22$. Test de Durbin-Watson :1.82.

Test de Ramsey : 1.4, Prob(0.23).

F-estadístico:2.45, Prob (0.0049).

Test de Breusch-Pagan : 0.92, Prob(0.51).

*Significativo al 95\%. **Significativo al 90\%. Entre paréntesis se muestra el error estándar. 
verdadera ventaja competitiva a través del incremento en la eficiencia de sus procesos y de la mejora de la calidad y grado de diferenciación que logre con sus productos.

\section{Conclusiones}

La estructura empresarial mexicana está compuesta en su mayoría por microempresas, las cuales son la principal fuente de empleo, ya que concentran 42 por ciento de la población ocupada. En los años recientes, estas unidades económicas se han convertido en un instrumento para compensar el estrechamiento del mercado laboral y la desocupación que las medianas y grandes empresas están generando con los recortes a sus plantillas laborales para enfrentar la caída en la demanda de sus productos, sobre todo en tiempos de crisis. Aunque la gran mayoría de estos emprendimientos surgen por necesidad, se encuentra que los microempresarios pueden fijar precios en sus productos por encima de los niveles de mercado, aun cuando sus procesos productivos sean de bajo valor agregado.

En este trabajo se identifica que los aspectos estratégicos del negocio y el ambiente de mercado son los factores endógenos que tienen mayor incidencia en la posibilidad de ejercicio de poder de mercado de las microempresas surgidas en entornos de marginación, mientras que las que menos están asociadas a este ejercicio son las cualidades de los trabajadores y del producto.

Uno de los principales retos para estos establecimientos es el de superar la trampa de la subsistencia, derivada de una demanda básica que los provee de un ingreso de corto plazo que favorece la descapitalización por el desgaste de los bienes de capital. Es indispensable fomentar mecanismos para que a pesar de sus restricciones productivas, estas microempresas mejoren la capacitación de sus trabajadores y sus prácticas de atención al cliente como espacio para innovar y competir en precio, sin sacrificar la calidad de sus productos. Esto les permitirá crear ciclos de mejora continua que ayuden a incrementar su rentabilidad, favoreciendo su incursión en nuevos mercados y logrando con ello superar su naturaleza de sobrevivencia.

Considerando el mercado como un proceso dinámico, son las variables asociadas a la gestión general del establecimiento las que permiten compensar muchas de las dificultades e ineficiencias de las microempresas y mejorar su nivel de ingresos. En el diseño de políticas públicas de fomento al desarrollo microempresarial se debe considerar el impulso de la calidad, innovación y eficiencia productiva que mejore la dotación de activos con los que producen. Lo anterior se puede conseguir mediante la implementación de programas novedosos y oportunos de capacitación, asistencia, marketing y financiamiento. 


\section{REFERENCIAS BIBLIOGRÁFICAS}

Almaraz, Ignacio (2013), Administración financiera como estrategia para lograr ventajas competitivas en las organizaciones, México, Editorial Fontamara, 288 pp.

Audretsch, David (2001), "Research Issues Relating to Structure, Competition, and Performance of Small Technology-Based Firms", Small Business Economics, vol. 16, pp. 37-51.

Audretsch, David y Yamawaki Hideki (eds.) (1991), Structure, Conduct and Performance, Nueva York, University Press, 437 pp.

Barla, Philippe (2000), "Firm size inequality and market power", International Journal of Industrial Organization, vol. 18, pp. 693-722.

Bikker, Jacob A.; Shaffer, Sherrill y Spierdijk, Laura (2012), "Assessing Competition with the PanzarRosse Model: The Role of Scale, Costs, and Equilibrium”, Review of Economics and Statistics, vol. 94, núm. 4, pp. 1025-1044.

Borch, Odd-Jarl y Forsman, Sari (1999), "The Competitive Tools and Capabilities of Micro Firms in the Nordic Food Sector-a Comparative Study", en S. O. Borgen (ed.), The Food Sector in Transition - Nordic Research, Proceedings of NJF-seminar núm. 313, Junio del 2000. NILF-Report 2001-2. Oslo, Norwegian Agricultural Economics Research Institute, pp. 33-50. (2004), "Competitive Positioning and Resource Configuration of Small Firm in a Mature Industry", en Thomas Cooney y Pasi Malinen (ed.), New Perspective on Firm Growth, $1^{\text {st }}$ Inter-Rent online publication, Europan Council for Small Business and Entrepreneurship, Turko.

Bresnahan, Timothy (1989), "Empirical Studies of Industries with Market Power", en R. Schmalensee y R. Willig (eds.), Handbook of Industrial Organization, vol. 2 (cap. 17), pp. 1011-1057.

Chamberlin, Edwars (1933), The Theory of Monopolistic Competition, Cambridge, Harvard University Press.

Clarke, Roger, (2000), Economía industrial, Ed. Colegio de Economistas de Madrid y Celestes Ediciones, Madrid.

Daniels, Lisa (1999), "The Role of Small Enterprises in the Household and National Economy in Kenya: A Significant Contribution or a Last Resort?", World Development, vol. 27 (1), enero, pp. 55-65.

Davies, Stephen; Seale, James; Mead, Donald; Badr, Mahmoud; Sheikh, Nadia y Saidi, Abdel (1984), "Small Enterprises in Egypt: A Study of Two Governorates", MSU International Development Papers, Working Paper, núm. 16, 
112 ECONOMÍA. TeORÍA y PRÁCTICA • Nueva Época, número 46, enero-junio 2017

East Lansing, Michigan State University, Department of Agricultural Economics, $100 \mathrm{pp}$.

Demsetz, Harold (1974), “Two Systems of Belief About Monopoly”, en Harvey Goldschmid et al. (eds.), Industrial Concentration: the New Learning, Boston, Little-Brown.

Elizing, Kenneth y Mills, David (2011), "The Lerner Index of Monopoly Power: Origins and Uses", American Economic Review, vol. 101, núm. 3, pp. 558-564.

Fariñas, José y Huergo, Elena (2003), "Profit Margins, Adjustment Costs and the Business Cycle: An Application to Spanish Manufacturing Firms", Oxford Bulletin of Economics and Statistics, vol. 65 (1), febrero, pp. 49-72.

Georgellis, Yannis; Sessions, John G. y Tsitsianis, Nikolaos (2005), "Self-Employment Longitudinal Gynamics: A Review of the Literature", Economic Issues, vol. 10, núm. 2, pp. 51-84.

Gross, Daniel (2011), La innovación como estrategia. Cuando renovar y cambiar conducen al éxito, Profit Editorial, Forbes, S. L., 107 pp.

Guarneros, Israel (2013), Más competitivas que pequeñas. Imagen corporativa a la medida de su empresa, México, Grupo Editorial Patria, 224 pp.

Hotelling, Harold (1929), "Stability in Competition”, The Economic Journal, vol. 39, núm. 153, pp. 41-57.

Huergo, Elena (2001), “El diagnóstico de poder de mercado en economía industrial: Una revisión de la literatura empírica española del siglo xx", Documento de Trabajo 0112 de la Universidad Complutense de Madrid.

Landa, Juan Pablo (2013), Flexibilidad interna e innovación en la empresa, Madrid, Editorial Dikynson, 355 pp.

Lerner, Abba (1934), "The Concept of Monopoly and the Measurement of Monopoly power", Review of Economic Studies, vol. 1, núm. 3, junio, pp. 157-175.

López, Alejandra; Contreras, Ricardo y Molina, Rubén (2013), La empresa familiar como objeto de estudio, México, Editorial Pearson Educación, 360 pp.

Mills, David y Schumann, Laurence (1985), "Industry Structure with Fluctuating Demand", American Economic Review, vol. 75, núm. 4, pp. 758-767.

Mungaray, Alejandro y Ramírez, Natanael (2004), "Subcontratación en microempresas y pequeñas empresas de Baja California", Frontera Norte, vol. 16, núm. 32, pp. 35-62.

Mungaray, Alejandro; Ramírez, Natanael y Michelle, Texis (2005), "Estructura de mercado y maximización de beneficios en las microempresas", Comercio Exterior, vol. 55, núm. 4, abril, pp. 316-321. 
Mungaray, Alejandro; Ramírez, Natanael; Aguilar, José G. y Beltrán, José M. (2007), "Poder de mercado en microempresas de Baja California", Problemas del Desarrollo, vol. 38, núm. 148, pp. 173-194.

Orlando, M. Beatriz y Pollack, Molly (2000), Microenterprises and Poverty: Evidence from Latin America, Washington D.C., Inter-American Development Bank.

Palacio, Juan I. (2002), "Política industrial activa para microempresas en el funcionamiento de los mercados: Una perspectiva Española", en A. Mungaray, J. I. Palacio y C. Ruiz-Durán (coords.), Potencial de la vinculación universitaria para una politica microempresarial: Una perspectiva comparada, México, M. A. Porrúa-UABC-uclm, pp. 37-68.

Parada, Elva Leticia (2013), La mujer empresaria en México, México, Editorial Pearson Educación, 280 pp.

Parkin, Michael y Esquivel, Gerardo (2006), Microeconomía: Versión para Latinoamérica, México, Pearson Educación, 584 pp.

Pollack, Robert (2005), "Bargaining Power in Marriage: Earnings, Wage Rates, and Household Production", NBER Working Paper No. 11239, Washington University.

Shama, Avraham (1993), "Marketing Strategies During Recession: a Comparison of Small and Large Firm", Journal of Small Business Management, julio, vol. 31, núm. 3, pp. 62-72.

Shultz, Theodore (1960), "Capital Formation by Education”, Journal of Political Economy, vol. 68, pp. 571-583.

(1971), "Investment in Human Capital", en Ronald Wykstra (ed.), Education and the Economics of Human Capital, Nueva York, The Free Press, pp. 23-41.

Stoft, Steven (2002), Power System Economics: Designing Markets for Electricity, Nueva York, IEEE Wiley Press, 496 pp.

Sylos, Labini (1966), Oligopolio y progreso técnico, Barcelona, Ediciones Oikos, $95 \mathrm{pp}$.

Vázquez-Barquero, Antonio (2005), Las nuevas fuerzas del desarrollo, Barcelona, Editorial Antoni Bosch, $192 \mathrm{pp}$.

White, Laurence (2013), "Market Power: How does it Arise? How is it Measured?", en Thomas y Shughart II (eds.), The Oxford Handbook in Managerial Economics, Oxford, University Press, pp. 31-65. 
